\title{
Amplified reflection, phase conjugation, and oscillation in degenerate four-wave mixing
}

\author{
Amnon Yariv and David M. Pepper \\ California Institute of Technology, Pasadena, California 91125 \\ Received March 3, 1977
}

\begin{abstract}
A number of new optical effects that result from degenerate four-wave mixing in transparent optical media are proposed and analyzed. The applications are relevant to time-reversed (phase-conjugated) propagation as well as to a new mode of parametric oscillation.
\end{abstract}

The purpose of this Letter is to examine briefly the consequences of a certain class of four-wave mixing. This mixing involves two intense pump waves $E_{1}$ and $E_{2}$ of the same frequency $\omega$ traveling in opposite directions and two "weak" waves $E_{3}$ and $E_{4}$ of the same frequency $\omega$, which also travel in opposition to each other but along a direction different from that of $E_{1}$ and $E_{2}$. The geometry is illustrated in Fig. 1.

The fields are taken as plane waves

$$
E_{i}(\mathbf{r}, t)=\frac{1}{2} A_{i}\left(r_{i}\right) e^{i\left(\omega t-\mathbf{k}_{i} \cdot \mathbf{r}\right)}+\text { c.c. }
$$

with $r_{i}$ the distance along $\mathbf{k}_{i}$. We thus have

$$
\mathbf{k}_{1}+\mathbf{k}_{2}=0, \quad \mathbf{k}_{3}+\mathbf{k}_{4}=0 \text {. }
$$

The nonlinear polarization coupling the waves is of the type ${ }^{1}$

$$
\begin{aligned}
& \begin{array}{l}
P^{(\mathrm{NL})}\left(\omega_{4}=\dot{\omega}_{1}+\omega_{2}-\omega_{3}\right) \\
\quad=\frac{1}{2} \chi A_{1} A_{2} A_{3} * \exp \left\{i\left[\omega_{4} t-\left(\mathbf{k}_{1}+\mathbf{k}_{2}-\mathbf{k}_{3}\right) \cdot \mathbf{r}\right]\right\}, \\
P^{(\mathrm{NL})}\left(\omega_{3}=\omega_{1}+\omega_{2}-\omega_{4}\right)=P^{(\mathrm{NL})}(w=w+w-w) \\
=\frac{1}{2} \chi A_{1} A_{2} A_{4} * \exp \left\{i\left[\omega_{3} t-\left(\mathbf{k}_{1}+\mathbf{k}_{2}-\mathbf{k}_{4}\right) \cdot \mathbf{r}\right]\right\} .
\end{array}
\end{aligned}
$$

We consider a medium with a nonlinear susceptibility $\chi$ occupying the space $0<z<L$. We are interested in the spatial evolution of waves $A_{3}$ and $A_{4}$ when subject to the pumping by $A_{1}$ and $A_{2}$.

We apply the standard ${ }^{2-4}$ methods of nonlinear optics to solve the wave equation

$$
\nabla \times \nabla \times \mathbf{E}+\frac{\epsilon}{c^{2}} \frac{\partial^{2} \mathbf{E}}{\partial t^{2}}=-\frac{4 \pi}{c^{2}} \frac{\partial^{2}}{\partial t^{2}} \mathbf{P}^{(\mathrm{NL})},
$$

and, heglecting the depletion of $A_{1}$ and $A_{2}$, obtain

$$
\begin{gathered}
\frac{\mathrm{d} A_{3}}{\mathrm{~d} z}=i \frac{2 \pi \omega}{c n} \dot{x} A_{1} A_{2} A_{4} * \exp \left\{-i\left(\mathbf{k}_{1}+\mathbf{k}_{2}-\mathbf{k}_{3}-\mathbf{k}_{4}\right) \cdot \mathbf{r}\right\} \\
=i \varkappa^{*} A_{4}^{*}, \\
\frac{\mathrm{d} A_{4}^{*}}{\mathrm{~d} z}=i \varkappa A_{3},
\end{gathered}
$$

where we used the fact that $\mathbf{k}_{1}+\mathbf{k}_{2}=0, \mathbf{k}_{3}+\mathbf{k}_{4}=0$,

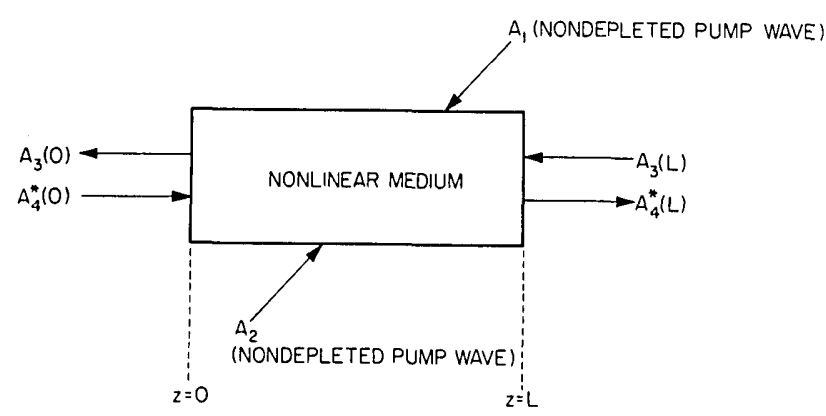

Fị. 1. Four-wave mixing geometry (assuming nondepleting pump waves).

took the $z$ direction as that of $\mathbf{k}_{4}$, and used the adiabatic approximation

$$
\left|\frac{\mathrm{d}^{2} A_{i}}{\mathrm{~d} z^{2}}\right| \ll\left|k_{i} \frac{\mathrm{d} A_{i}}{\mathrm{~d} z}\right| .
$$

The complex coupling constant $x$ is given by

$$
x^{*}=\frac{2 \pi \omega}{c n} \chi A_{1} A_{2} .
$$

If we specify the complex amplitudes $A_{3}(L)$ and $A_{4}(0)$ of the two weak waves at their respective input planes ( $z=L, z=0$ ), the solution of Eq. (5) is

$$
\begin{aligned}
& A_{3}(z)=\frac{\cos |\varkappa| z}{\cos |\varkappa| L} A_{3}(L)+i \frac{\varkappa^{*}}{|\varkappa|} \frac{\sin |\varkappa|(z-L)}{\cos |\varkappa| L} A_{4} *(0), \\
& A_{4}^{*}(z)=i \frac{|\varkappa|}{\varkappa^{*}} \frac{\sin |\varkappa| z}{\cos |\varkappa| L} A_{3}(L)+\frac{\cos |\varkappa|(z-L)}{\cos |\varkappa| L} A_{4}^{*}(0) .
\end{aligned}
$$

An especially interesting case is that of a single input $A_{4}(0)$ at $z=0$. In this case the reflected wave at the input $(z=0)$ is

$$
A_{3}(0)=-i\left(\frac{x^{*}}{|x|} \tan |x| L\right) A_{4}^{*}(0)
$$

while at the output $(z=\mathrm{L})$,

$$
A_{4} *(L)=\frac{A_{4} *(0)}{\cos |x| L} .
$$




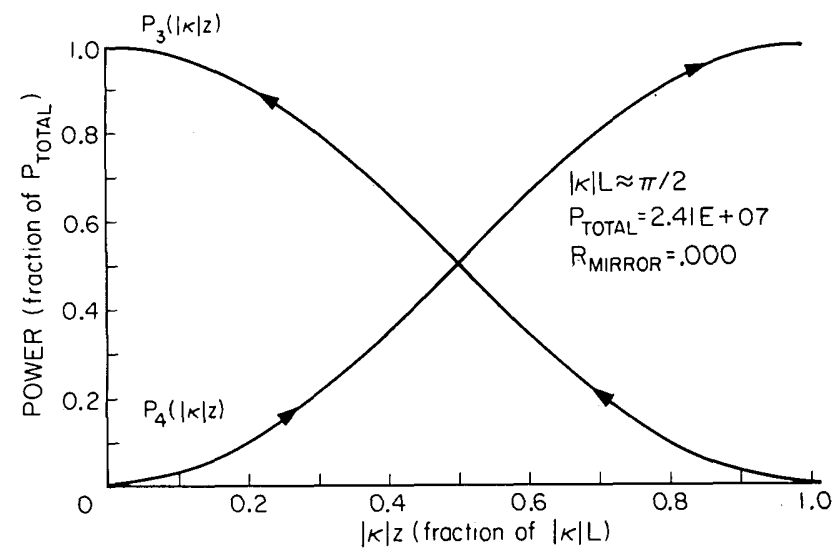

Fig. 2. Intensity distributions of the input power $\left(P_{4}\right)$ and the conjugate field power $\left(P_{3}\right)$ as a function of $|\varkappa| z$ near oscillation condition $(|x| L=\pi / 2)$.

We note that for $\pi / 4<|x| L<3 \pi / 4$,

$$
\left|A_{3}(0)\right|>\left|A_{4}(0)\right|
$$

and the reflected wave amplitude exceeds that of the input. The device acts as a reflection amplifier. The transmitted wave $\left|A_{4}(L)\right|$ exceeds the input $\left|A_{4}(0)\right|$ under all conditions.

When

$$
\begin{gathered}
|\varkappa| L=\pi / 2, \\
\frac{A_{3}(0)}{A_{4}{ }^{*}(0)}=\infty, \quad \frac{A_{4}^{*}(L)}{A_{4} *(0)}=\infty,
\end{gathered}
$$

which corresponds to oscillation. The four-wave mixing process, in analogy to a backward parametric oscillator, ${ }^{5}$ is capable of oscillation without mirror feedback.

The field distribution near the oscillation condition is illustrated in Fig. 2.

Phase conjugation and amplification are also possible under conditions of three-wave backward parametric amplification in crystals. ${ }^{5}$ A fundamental difference between the three-wave mixing process and the fourwave process described here is that the former depends on phase matching and the latter does not [note that the sum $\mathbf{k}_{1}+\mathbf{k}_{2}-\mathbf{k}_{3}-\mathbf{k}_{4}$ in the exponents of Eq. (5) is identically zero]. This difference leads to a number of important practical considerations: (1) The three-wave mixing process is limited in practice to crystals of very large birefringence and to large ratios of input and output frequencies-both conditions being necessary to overcome the "unnatural" phase matching condition. ${ }^{5}$ (2) Because of its freedom from the need for phase matching, the four-wave phase mixing process can be used to amplify wavefronts of arbitrary complexity.

To prove the last statement we take the input field $A_{4}$ in the form of an arbitrary superposition of plane waves using a Fourier expansion

$$
\begin{aligned}
A_{4}(x, y, z) & =\int A_{4}\left(\mathbf{k}_{\perp}, z\right) e^{i \mathbf{k}_{\perp} \cdot \mathbf{r}_{\perp} \mathrm{d}^{2} \mathbf{k}_{\perp}} \\
& =\int \dot{A}_{4}\left(-\mathbf{k}_{\perp}, z\right) e^{-i \mathbf{k}_{\perp} \cdot \mathbf{r}_{\perp}} \mathrm{d}^{2} \mathbf{k}_{\perp},
\end{aligned}
$$

while the output field is taken as

$$
A_{3}(x, y, z)=\int A_{3}\left(\mathbf{k}_{\perp}, z\right) e^{i \mathbf{k}_{\perp} \cdot \mathbf{r}} \mathrm{d}^{2} \mathbf{k}_{\perp} .
$$

Choosing the pump beams $A_{1}$ and $A_{2}$ as plane waves traveling in opposition to each other along the same arbitrary direction,'we obtain by substituting Eqs. (12) and (13) in the wave equation (4) and after some rearrangement

$$
\begin{aligned}
& \frac{\partial}{\partial z} A_{3}\left(\mathbf{k}_{\perp}, z\right)=-i \frac{\lambda k^{2} \perp}{4 \pi} A_{3}\left(\mathbf{k}_{\perp}, z\right) \\
& \frac{\partial}{\partial z} A_{4} *\left(-\mathbf{k}_{\perp}, z\right) \\
& =-i \frac{\lambda k^{2} \perp}{4 \pi} A_{4} *\left(-\mathbf{k}_{\perp}, z\right)+i \varkappa A_{3}\left(\mathbf{k}_{\perp}, z\right), \\
& +i \varkappa^{*} A_{4}^{*}\left(-\mathbf{k}_{\perp}, z\right),
\end{aligned}
$$

where $x$ is given by Eq. (6) and $k=(\omega / c) n$.

A specified input phase front at $z=0$ amounts to specifying $A_{4} *\left(-\mathbf{k}_{\perp}, 0\right)$. Since no mixing takes place at $z>L$, we take the reflected field $A_{3}\left(\mathbf{k}_{\perp}, L\right)$ to be zero at the output $z=L$. With these boundary conditions, the solution to Eq. (14) is

$$
\begin{aligned}
& A_{3}\left(\mathbf{k}_{\perp}, z\right) \\
& =i e^{-i \lambda_{\perp} 2 z / 4 \pi}\left(\frac{\varkappa^{*}}{|\varkappa|}\right) \frac{A_{4}\left(-\mathbf{k}_{\perp}, 0\right)}{\cos \varkappa L} \sin |x|(z-L), \\
& A_{4} *\left(-\mathbf{k}_{\perp}, z\right)=e^{-i \lambda_{\perp} 2_{z} / 4 \pi} A_{4} *\left(-\mathbf{k}_{\perp}, 0\right) \\
& \frac{\cos |\varkappa|(z-L)}{\cos |\varkappa| L} \cdot
\end{aligned}
$$

At the input to the nonlinear medium, $z=0$, we thus have

$$
A_{3}\left(\mathbf{k}_{\perp}, 0\right)=-i\left(\frac{\varkappa^{*}}{|\varkappa|} \tan |\varkappa| L\right) A_{4}{ }^{*}\left(-\mathbf{k}_{\perp}, 0\right),
$$

which shows that the individual plane wave components of the arbitrary wavefronts behave as in the plane wave case except that each $\mathbf{k}_{\perp}$ component of the output beam $A_{3}$ couples directly to the $-\mathbf{k}_{\perp}$ component of the input wave $A_{4}$. It is now a straightforward, though formal, procedure to show, using the uniqueness property of linear parabolic differential equations, that the relation of Eq. (16) in conjunction with Eqs. (4) and (13) signifies

$$
\begin{aligned}
A_{3}(x, y, z & <0) \\
& =-i\left[\left(\frac{x^{*}}{|x|} \tan |x| L\right)\right] A_{4}^{*}(x, y, z<0),
\end{aligned}
$$

so that an arbitrarily complex incident wavefront $A_{4}$ gives rise, at $z<0$, to a reflected and amplified field $A_{3}$, which is everywhere the complex conjugate of $A_{4}$. The complex conjugate nature of the reflected wave is important in phase-distortion correction.6,7 Equation (17) in the limit of $|x| L \ll 1$ has been obtained by Hellwarth. ${ }^{8}$ His analysis, which was limited to $|x| L \ll 1$, does not reveal the possibility of amplification of oscillation.

We have shown above that oscillation results when $|x| L=\pi / 2$. This condition is most likely to be satisfied when the waves $A_{3}$ and $A_{4}$ are parallel to the input waves $A_{1}$ and: $A_{2}$, since this is the direction of maximum beam overlap. This, however, is the direction of least interest, since the "output" waves $A_{3}$ and $A_{4}$ will be 


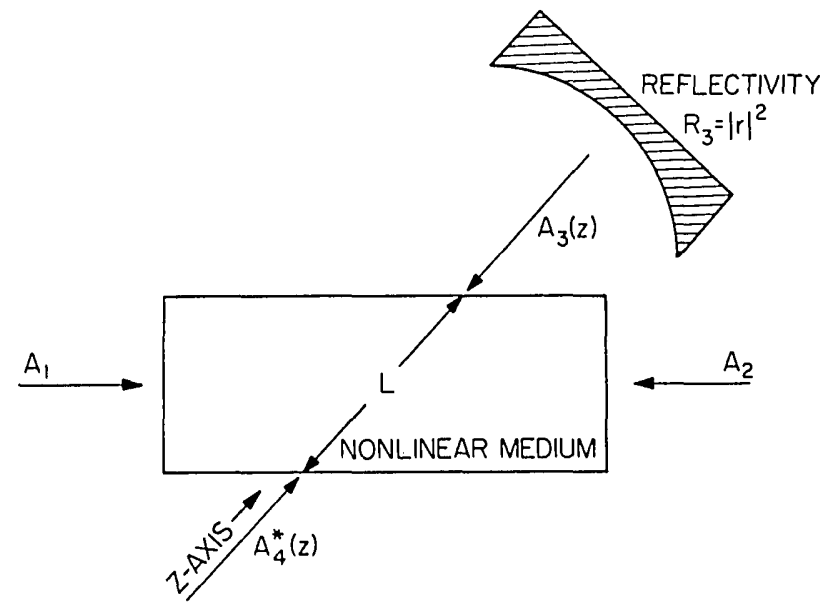

Fig. 3. Four-wave mixing utilizing external mirror of reflectivity $R_{3}$, which provides preferred direction for oscillation.

degenerate in their directions as well as their frequencies with the "pump" waves $A_{1}$ and $A_{2}$.

To solve this problem we may add a single reflector along some arbitrary direction, as shown in Fig. 3. It follows directly from Eq. (7) and the boundary condition imposed by the mirror that the oscillation condition in Eq. (11) is now replaced by

$$
|\varkappa| L=\tan ^{-1}(1 /|r|),
$$

where $|r|^{2}$ is the reflectivity of the mirror. For a mirror with near-unity reflectivity, oscillation occurs at $|x| L$ $=\pi / 4$, which is lower by a factor of 2 than that of the no-mirror case. The presence of an external mirror thus defines the path of lowest threshold.

In summary: We have a new class of optical effects that can be achieved by degenerate four-photon mixing. These include amplified reflection, transmission, phase conjugation, and oscillation. A more detailed analysis as well as numerical considerations will be given in a separate publication.

The authors acknowledge fruitful discussions with P. Yeh and P. Agmon. One of the authors (DMP) is thankful for the support granted by the Hughes Aircraft Company.

David M. Pepper is a Hughes Research Laboratories Doctoral Fellow.

\section{References}

1. P. D. Maker and R. W. Terhune, Phys. Rev. 137, A801 (1965).

2. N. Bloembergen, Nonlinear Optics (Benjamin, New York, 1965).

3. A. Yariv, Quantum Electronics (Wiley, New York, 1975).

4. J. J. Wynne and G. D. Boyd, Appl. Phys. Lett. 12, 191 (1968).

5. S. E. Harris, Appl. Phys. Lett. 9, 114 (1966).

6. B. Zeldovich et al., Phys. JETP Lett. 15, 109 (1972).

7. A. Yariv, Appl. Phys. Lett. 28, 88 (1976); also, Opt. Commun. (to be published).

8. R. Hellwarth, J. Opt. Soc. Am. 67, 1 (1977). 